DE DE GRUYTER

OPEN

G

BULGARIAN ACADEMY OF SCIENCES

CYBERNETICS AND INFORMATION TECHNOLOGIES • Volume 15, No 5

Special Issue on Control in Transportation Systems

Sofia $\bullet 2015$

Print ISSN: 1311-9702; Online ISSN: 1314-4081

DOI: $10.1515 /$ cait-2015-0016

\title{
Framework for an Autonomic Transport System in Smart Cities
}

\author{
Jörn Schlingensiepen ${ }^{1}$, Rashid Mehmood ${ }^{2}$, Florin Codrut Nemtanu ${ }^{3}$ \\ ${ }^{1}$ Technische Hochschule Ingolstadt, Germany \\ ${ }^{2}$ King Khalid University, Abha, Kingdom of Saudi Arabia \\ ${ }^{3}$ POLITEHNICA University of Bucharest, Romania \\ Emails: joern@schlingensiepen.com R.Mehmood@gmail.com florin.nemtanu@ieee.org
}

\begin{abstract}
Today the concept of smart cities is discussed in scientific society and politics. A core function of smart cities is transportation. This paper gives a short overview on the concepts for Intelligent Transport Systems (ITS) for smart cities and proposes a framework for the design of an autonomic transportation system that provides personalized mobility services to its users in a smart city setting. The transportation poses extreme environment for ICT systems due to fast moving vehicles and users, requiring real-time acquisition and high performance processing of large scale data, and rapidly changing communication networks topologies and node densities. The aim of this paper is to propose a framework that will act as a reference for the design of future transportation systems that are able to cope with the ever rising system complexities and users' demands. Therefore, a backbone system providing information at different levels was designed following the principles of a corporative ICT that were proposed in [1]. The framework fulfills the main requirement providing suitable information about the local decision engines in vehicles and infrastructure interacting in smart cities traffic systems.
\end{abstract}

Keywords: Autonomic Road Transport System (ARTS), COST-ARTS, autonomic ICT systems, Intelligent Transport System (ITS), service assessment, service discovery, corporative ICT architecture.

\section{Introduction}

The concept of smart cities assumes that the functions of the cities are provided in a smart way to their citizens. The common understanding of "smart" is:

- reducing the amount of resources used to provide functions to citizens, 
- focusing on services - not on infrastructure - to provide these functions,

- integration of services, respectively service providers to gain a suitable and pleasant user experience.

On the other hand, there are 6 main characteristics of a city, defined in [2], which are placing the city in the smart zone: smart mobility, smart economy, smart environment, smart people, smart living and smart governance.

This approach causes some socio-technological issues. "Smartness" will cause a higher grade of automation like autonomous systems and the question of reliability in these systems is not clear (there is not much public discussion about this) - today the UN-Convention on Road Traffic Vienna [3] permits autonomous vehicles in general - and of course, the question of privacy has to be discussed.

Besides these legal and sociological issues we need to think of cities infrastructures in a new way. This meansthe development of new business cases and of course, a new definition of the infrastructure provided by public and private owners. In this paper we will focus on the main public function of cities: Transportation.

Intelligent Transport Systems (ITS) can help optimizing the overall system. Since mass transportation does not fulfill the demands in individual/personal transport, future transport systems will be multimodal, combined transport systems including cars, vans and trucks, as well as buses, trains and other public transport vehicles. The actors in these systems will make their own local decisions by the human passengers or local decision engines. Therefore, the vehicles and passengers need to be integrated in these systems by mobile devices/controllers (on board units), so new requirements towards the vehicles and traffic management systems are coming up. The spreading of incorporated units doing local decisions brings the use of distributed software system architecture. So the main purpose for this backbone is providing current data to the local decision engines in a fast and reliable way. Autonomic Road Transport Systems (ARTS) face these problems and provide a new approach for establishing the transportation networks.

\subsection{Preconditions}

According to [4] one of the most important factors for an Intelligent City is an open, interoperable and scalable platform that provides intelligent infrastructure functionality as a service and allows optimal resource management. Fig. 1 shows the main aspects of the open interoperable infrastructure for a smart city.

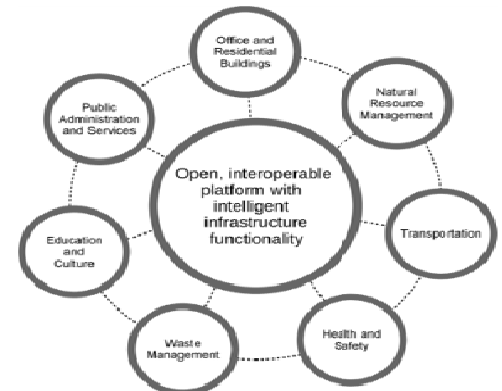

Fig. 1. Main aspects of open interoperable infrastructure for a smart city [4] 


\subsection{Smart travel choice in a smart city}

The concept of smart cities needs flexible partnership between the public and private sectors, as well as diverse industries, such as telecommunications, energy providers, manufacturers and suppliers that ensure improvements in mobility, energy consumption, governance and social cohesion in European cities [5]. At present the modal split of the transport in Europe is dominated by the passenger cars $(73.4 \%$ compared to just $1.4 \%$ combined for the tram and metro). According to the World Health Organization, about 40 million people in the 115 largest cities in the EU are exposed to air exceeding WHO air quality guideline values for at least one pollutant [6].

Autonomous vehicles, as a consenquence of environmental travel approach, within a decade or two will probably enable the doubling of the highway capacity and in 2040-ies the autonomous vehicles are likely to represent approximately 50\% of the new vehicles sales, $30 \%$ of the total vehicle fleet and $40 \%$ of the total vehicle travel [7]. Even now the basic cars have 30 or more computers (in some luxury cars, more than hundred - the electronics represents $15-20 \%$ of their cost) and we must make good use of it in improving the city environment [8].

Two main directions of the action have been aproached by USDoT, one in terms of autonomous vehicles - the big impact they will have on safety, mobility and the environment revealed by tests shows a range of fuel reduction between 4.5 and $25.1 \%$ in cars and $2.4-15.3 \%$ for trucks [9]. The second one, Real-Time Information Synthesis (AERIS) shows that the application itself can provide average fuel savings of $5-10 \%$ per vehicle (annual savings of 170 USD for cars and 280 USD for SUVs, based on vehicles driving 8000 miles per year on arterial roads [10]). Big energy savings can be done also by using smart street lighting - in Edmonton (Canada) the streetlights account for $21 \%$ of the city power consumption and now they are trying to reduce it by 40 up to $50 \%$ [11].

\subsection{Strategy for smart sustainable urban transport}

The negative impact of the transport is estimated annualy at almost $1 \%$ of EUs GDP (100 billions Euro) and according to [12] throughout Europe, increasing of the traffic in urban areas leads to permanent congestion.

2011 GER report on transport [13] states that "a green, low carbon, transport sector can reduce the greenhouse gas emissions by $70 \%$ without major additional investment", and $0.16 \%$ of global GDP invested in boosting the public transport infrastructure will reduce the volume of the road vehicles by a third until 2050 .

The Commission is very active in defining urban transport policies (i.e., EU Thematic Strategy on the Urban Environment [14] sets up a number of measures and one is the adoptation of sustainable urban mobility plan). More information and definition of the challenges on the way towards smart sustainable urban transport can be found in [12]. 


\subsection{Objectives for a smart transport system}

\subsubsection{The political agenda}

The fourth priority area of the European ITS Directive [15] is focused on linking the vehicles with the infrastructure based, especially, on open in-vehicle platform, as well as cooperative systems. This means integration between the vehicles and the infrastructure. The Autonomic Road Transport Systems (ARTS) could be the key to solve traffic and mobility problems in cities (Urban ITS Expert Group has already elaborated a set of guidelines oriented on ITS for urban areas, i.e., Guidelines for ITS Deployment in Urban Areas - Multimodal Information [16]).

\subsubsection{User benefits}

The user experience while using a smart city service has to be designed in a way that enables the user to get a better service with respect to his needs, fulfilled with less effort/time consumption and minimum of interaction needed, that leads to the main issues to be addressed:

- resources need to be allocated predictively,

- allocation of the resources has to follow the overall benefit,

- peaks in needs have to be eliminated,

- the overall system needs to be fault tolerant, it has to work even if there are disfunctions in some areas.

\subsection{Influencing the traffic}

To allow an efficient traffic management, the current traffic situation and operating grade has to be captured to allow predictions of future situations to hedge decisions made by users, operators and decision engines.

All described aspects of smart cities concerning Smart Transport Systems require good understanding of the mechanisms. The traffic of independent and controlled vehicles is organized and can be influenced by managed infrastructure and service elements like traffic lights, special trains, etc. A pre-condition for a Smart Transport System is the automated decision system to control these influencing elements. The main issue while implementing this kind of systems is providing a good basis for decision to the decision engines. The best basis for decisions is knowing the current state of the overall system. That is why we have to focus on the data aquistion and the networks transporting information about the current situation in the field.

The decisions in the vehicles are made by the on board support decision engine or the driver (see Fig. 2). Today and in the nearer future for legal reasons and reasons of the users' acceptance, all decisions at all levels will be made by the driver, so the information of ITS is incorporated in the decision making process as suggestions, provided to the driver by a mobile device called adviser. Studies of the University of Twente have shown that the drivers' trust in the advice of the system is an essential requirement and this confidence is often absent [17]. The drivers make decisions (e.g., choosing lanes, speed adjustment) on the basis of personal experience. Therefore, the effect of not trusting the advice is boosted, because in 
traffic systems the results of decisions that are inauspicious for the overall system are not hurting the decision maker, but the other road users.

Fig. 3, derived from [18], shows some examples for actions, respectively decisions made at different levels, details about this can be found in [18].

\section{Approach}

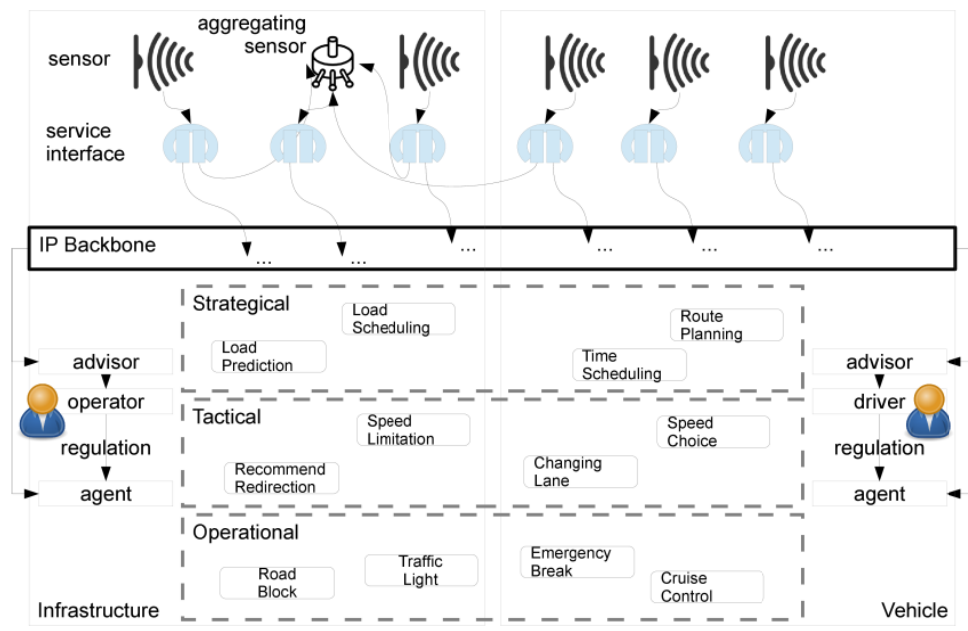

Fig. 2. Skeleton of a smart ICT including two sites (infrastructure and vehicle) a backbone and a set of data providers implementing the same interface

Based on [18] we propose the architecture shown in Fig. 2 (bottom half). As it can be seen in this figure, the ITS has the general structure of a distributed ICT system. The separation between the vehicles as moving parts and the infrastructure as fixed parts, is common. On both sites different information as data about the current state (mainly collected by sensors) and plans (input by users and operators) is collected. Based on this information, it is possible to derive control factors for the overall system. Therefore, this information must be brought together and evaluated. Today a general subdivision of communication in a Vehicle-to-Vehicle (V2V) and Vehicle-to-Infrastructure (V2I) is used. The main goal of the overall system is fulfilling the plans of the users (road users and traffic system operators) while using minimum of resources. Based on the aggregated data, both the infrastructure and the vehicle make predictions of the future state of the road and make local or global decisions. These decisions are made by the road users, by operators that may get recommendations from an adviser or decision engines and can be assigned levels refering to different time horizons: long-term planning (strategic), medium-term behaviour (tactical) and direct action/reaction (operational).

As seen in Fig. 2, the plans of the infrastructure provider are optimizing the utilization grade of the infrastructure and allow emergency prioritization on demand. Therefore, a model of the overall system (including the vehicle behaviour) and a statistical component, using machine learning (Stat-Com) are used to generate 
the load prediction for all street sections. This prediction engine has learned from the past and is fed by the current state of the overall system. The load prediction is written back to the backbone and used by the components on the vehicle site to derive local decisions.

On a vehicle site we have individual plans like reaching the route target, saving fuel and time. These plans influence the local decisions. By providing these plans to the network, the prediction of the infrastructure gets more precise. As an example for local decisions on a vehicle site the choice of speed, lane and route can be named. These decisions will be made on the basis of the local prediction of Lane Limitations and Route Limitations. For example, reducing/increasing of the speed might be reasonable if the best route has limitations that will be gone/arisen sometimes later and an alternative route is not suitable.

In order to allow all these predictions, information about the current state has to be collected from the field. On the infrastructure site this information is collected by internal sources, like traffic observers but also external sources, like weather conditions (including forecast) or a passenger counter from public transport systems. On the vehicle site, information about the current state like speed and global position, and also information about the environment like local position (lane, direction), and distance to other vehicles is collected to allow a proper model of the current situation, e.g., traffic load.

\section{Requirements to the architeture}

The first requirement towards the future systems is at a communication network level. The technologies chosen must enable the exchange of traffic information between the system components from different vendors in a kind of open Peer-toPeer (P2P)-system. Users can join and leave the network at any time. The components of the infrastructure assume different roles than those of the vehicles. Such kind of asymmetrical P2P systems are already sufficiently investigated (compare [1]), therefore the challenges to the system design can be easily derived. Following the already known problems from peer-to-peer-systems must be considered in the case given in [18]:

- V2V trust: In P2P-systems information is provided by every peer. Therefore, the peers need an internal model of evaluating the trustability of the provided information.

- V2I trust: The infrastructure provided by local authorities may help solving the problems of trust by providing mechanisms for collecting and verifying the information and prognosis.

- V2V pollution: A common problem in open P2P-Systems is the so called pollution $[19,20]$, where malicious peers may send manipulated data to affect the overall system and change its behaviour in order to earn a benefit or just to create confusion.

- V2V/V2I anonymity: In contrast to the requirements of trust, the road user requires a level of anonymity that ensures that the individuals are not tracked by 
authorities or other parties. Especially, in case of transmitting the user' plans via the system to prediction engines, privacy is a recommended function.

\section{Technology}

At this point it is obvious that reliability of the data is the essential and most important requirement to the ITS. Therefore, we propose in [18] the usage of the principles of autonomic systems. Establishing an autonomic system gives some great advantages in increasing the reliability of the overall systems. The term autonomic is a biological metaphor adopted by IBM to describe the desired properties of future complex IT systems, proposed as a highly advanced approach to deal with the problems of delivery and maintenance of increasingly complex systems. It is hypothesized that systems with autonomic capabilities will help alleviate many of the problems associated with life cycle management of complex systems. The autonomic systems embody self-assessment and self-management abilities that enable the system to assess its own state, then adapt or heal itself in response to this assessment. The interface between the system and the owner is set at a very high level: the owner sets out the goals, policies or service levels that the system must follow, and the system translates them into its system functions resulting in a change of the behaviour. IBM defines four areas of autonomic functions: (i) self-configuration through automatic configuration of components; (ii) self-healing through automatic discovery and correction of faults; (iii) self-optimization through automatic monitoring and control of resources to ensure the optimal functioning with respect to the defined requirements; and (iv) self-protection through proactive identification and protection from arbitrary attacks. We define autonomification as the process of transforming an entity (a service, system or infrastructure) into an autonomic entity, such that the process infuses autonomic properties into an entity causing the entity to become an autonomic entity. In terms of an ITS this influences two aspects of the system: Components design and architecture of the P2P-System. The components need to be implemented in a way that includes not only posting and consuming of data but also self-assessing and giving additional data about the own state and trustability of the measured data. Today's systems process only measured data with high trustability; in case of harvesting several data from many different measuring entitieș as done in our proposed system we have to add the capability of processing unsure data, that will lead to a more valid picture of the current system state.

The architecture of an autonomic ITS has to respect the fact, that the different components in the system are owned, run and configured by different stakeholders with different interests.

\section{System design and general architecture}

On the basis of the existing research approaches as the one given in [1], a general architecture for ITS to derive requirements for future vehicles and to identify issues 
was developed. Detailed description of this architecture can be found in [18]. In general, ITS backbones have to be considered as distributed ICT systems, dealing with all benefits and problems of these systems emerging from heterogeneous user groups, heterogeneous devices and the leak of confidence and reliability resulting from the fact that everybody can join and leave the system at any time.

\subsection{Run time environment}

Today's understanding of distributed ICT is running components in a cloud. In this concept every component like data storage, computing facilities, algorithms is seen as a service. So different software vendors and hosting providers are able to offer their various products in a common run time environment. The users benefit from this, because they need not to run their own infrastructure and the providers benefit because the utilization grade of the infrastructure is maximized.

Usually the different components are implemented as services, meeting a contract or service description by implementing an interface, so they can be replaced by alternative implementations. To allow the service consumers find a suitable service, the provider's services descriptions and service instances are listed in directories called service registry, and can be found using a lookup function provided by a service broker $[21,22]$.

The most common understanding of the services in this so called service oriented architecture is treating the service layer as a set of functions called from different systems. Samples for calls are handing over a business process to another system, using a software component to do some calculations, get information from a database or sensor, etc. At an implementation level this leads to the limitation the services to provide functions that a service consumer can call and get some result, as shown in Fig. 4, Synchronous and Polling. Today's very popular implementations of SOA using web services show this concept. In fact, most people use the web service and SOA as synonymous.

To allow integration of the traffic sensors and other data providers in SOA environment and providing capabilities of continuous data processing, we prefer using a pristine definition of the service orientation that allows understanding the sensors as a service, allows definition of the data processing chains and thus allows providing current data to decision engines in an almost real time way. These services and the network need to follow the principles given below:

- the consumers must be able to subscribe/unsubscribe the data collected by a sensor or any other data provider,

- the sensor or data provider as a service will emit collected data on registration or level of change,

- the network infrastructure will transport the collected data to subscribers in a fast and reliable way.

Based on these principles we propose the definition of a service interface for a general data provider. On this base we define a new sensor type - the aggregating sensor. These sensors collect information from other sensors locally and provide a higher level of information, e.g., combination of several counters generate information about the load of a street section. 


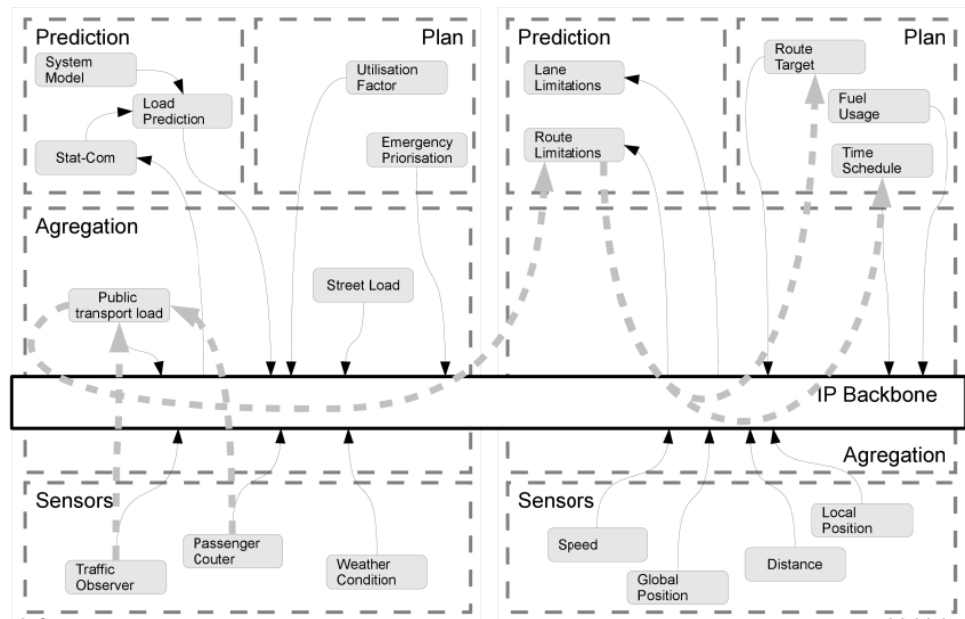

Fig. 3. Aggregation of data provides higher level information to allow better predictions [18]

Other components like prediction engines also implement this interface and provide their results as data to the network. Fig. 3 shows how this aggregation works at different levels of data acquisition and consumption.

Since the sensors and any data providing components are encapsulated as services, these components can be easily changed without effecting the overall system. There is also no need to implement different kinds of data consumers since the basic sensors, aggregation sensors and all other components provide the same interface (see Fig. 2, upper half). Since all data is available in the network, this setup allows implementation of the local decision engines, but since every stake holder or network member may provide data and the members join and leave the network continuously, we set the following requirements to the backbone:

- the local decision engines must be enabled to find the data they need by the providing component,

- the local decision engines must be able to judge the received data as reliable.

\subsection{Corporative ICT system}

The main problems of trust and pollution within a P2P system can be solved, using the concepts of a corporative ICT system [1] that are fulfilling these requirements: the service self-description, the service discovery, the service assessment and the asynchronous routable function call allow to build up a system meeting the described requirements. In this paper we can give only a short description, for further details refer to [1].

\subsubsection{Asynchronous routable function call}

In the sense of corporative ICT architecture means calling a service without getting the answer as a response. Fig. 4 illustrates the difference in the calling concepts. The direct call concept says the service provider sends the answer as a reply to the 
request immediately. In practice the lower layer data connection is kept to carry the answer. The polling call concept says that if the service provider needs more time to provide an answer, it replies by NOP (Not OPerated). In this case the client tries to get the answer by sending the poll requests. If the request is not processed it returns a NOP, otherwise the answer is delivered. The asynchronous function call means the client sends the request without expecting the result immediately, and the service provider sends the answer to the client after processing the request without any further polling. This concept presupposes an end to end connection and this concept allows sending an answer from a service provider that was not called. Thus routing of requests can be established.
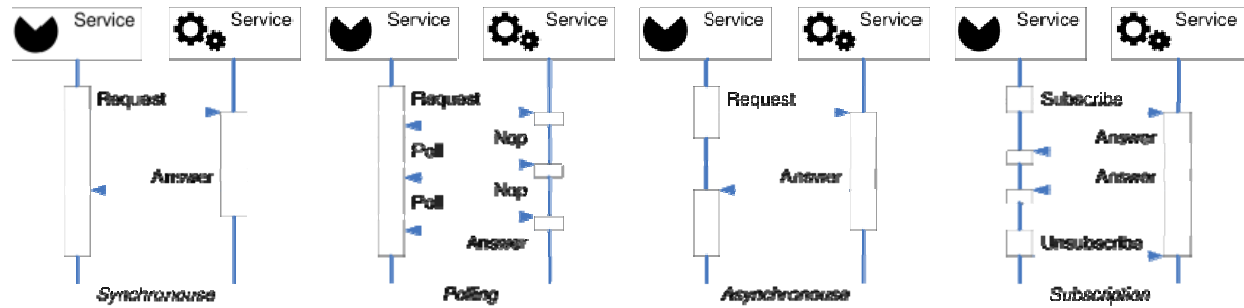

Fig. 4. Illustration of the direct call concept, polling call concept, asynchronous routable function call and subscription call concept

\subsubsection{Service discovery}

The discovery of services in a corporative ICT is done by an overlay network established using a Distributed Hash Table (DHT). On accessing a service, the Service Discovery component identifies the nodes offering the service. The client creates a message defining the required information and subscribes it to this node. If this node is not able to fulfill the requirements, the request for subscription is passed to another node (Fig. 5, steps 1-5).

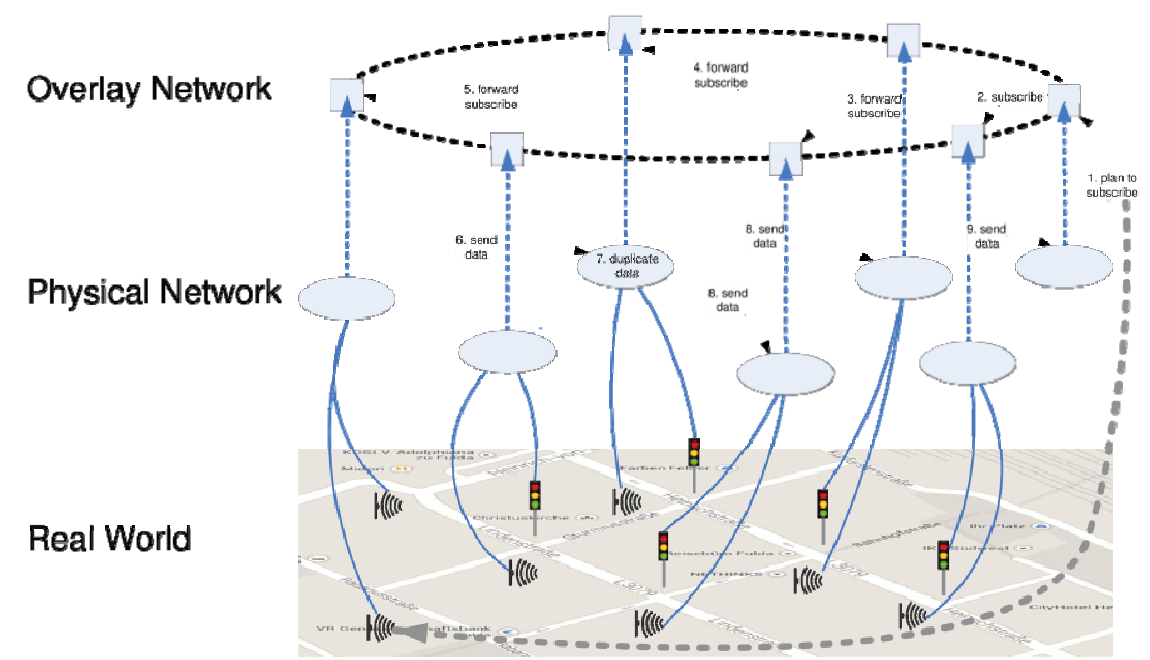

Fig. 5. Service discovery by routing a subscription request through an overlay network, data distribution by using multicast of IPv6 network 


\subsubsection{Service assessment}

Since the data providers are made available by different stakeholders, the data may be not trustable. Corporative ICT systems use the concept of service assessment to solve this problem. Therefore, each node provides a service self-description and each node is assigned to different trust zones. Based on this, the client can calculate the level of trustworthiness, details on this can be found in [1]. Since the infrastructure will be provided by public authority, the corporative ITS will be a hybrid P2P, where the so called super-peers will have higher credibility and provide the core functionality.

\subsection{Network layer}

Fault tolerance can be reached by placing the entity making operational and tactical decisions close to the actor [18], because this allows the actors in the system to be autonomous, so they are able to act, even if parts of the overall system are gone.

In our scenario this means that the vehicles, traffic lights, etc., must be able to gain all the information they need to make proper decisions. To ensure the function of the overall system, some supervising nodes may overrule the local decisions, but in case these are not longer available, or in case of danger, the local decision engine takes it over. Since the different components of the system will be produced by different vendors, the data transport has to be done in a standardized way.

To ensure simple and fault tolerant transport and distribution of the information, we propose a network based on IPv6. This protocol has three features ensuring a simple, safe and lightweight way of data transmission meeting the described demand:

- First of all an IP is designed to establish the networks between components from different vendors, it is a well accepted standard and allows integration of the new transmission technology without the need to change the software stack.

- Second IP is designed to ensure data transmission using different routes at runtime; this allows using the IP in an environment where the network topology is changing. Today's IP network components are able to reconfigure the routing if a network link is gone or a node is offline.

- Third feature of IPv6 allowing data transmission for our purpose is Multicast. Multicast allows sending information to many receivers. These receivers need to subscribe in a reserved address range. The infrastructure is able to route and duplicate the data packages to ensure multicast without wasting bandwidth.

Using IPv6-Multicast allows the use of several functions that help in establishing a reliable communication backbone for distributing data in the system. Fig. 5, Steps 6-9 shows the data distribution using multicast.

To ensure reliability on a physical layer, we propose the installation of a physical backbone that connects all components of the infrastructure. This fallback network must be connected to the public Internet via several gateways to allow transparent routing. Using this setup allows other stakeholders like logisticians, individual drivers, autonomous vehicles, pedestrians, etc., to subscribe the sensor data so as to feed their local decision engines. 
Using IPv6 multicast allows the integration of different components from different vendors. Since the current standards in networking in public transport and traffic management are based on IP (e.g., [23]), this will be a good approach to reuse the current infrastructure.

\section{Conclusion}

Using the described methods and concepts allows establishing a P2P-OverlayNetwork on the current networks. The chosen approach which uses an industry standard IPv6 with transparent routing and multicast, allows easy extension of this network to a public one to allow users joining the network by using their personal device, like a smart phone or a satellite navigation system of a vehicle or future personal devices that are carried by the user or will be fixed at a place or vehicle. Beside the personal devices, the components of the infrastructure like traffic management systems, traffic light systems, trains and data management systems of the service providers like a public transport provider, taxi associations and others can be integrated in the overlay network easily.

Since these systems are very heterogeneous, the future system will be asymmetric and may follow the approach of corporative IT-Systems [1], which enables using service oriented architecture of a cloud on the basis of a P2P-Overlay-Network. To define and standardize the architecture of a future system, the following tasks will be needed:

- Standardization of sensor profiles that allows choosing the proper sensor for a current demand,

- Standardization of the overlay network including protocols to enter and leave the network, provide and consume data/information by subscribing sensors/data providers that respect privacy,

- Standardization of the services in a network, including definitions how to exchange location data and types of information.

- Standardization of the quality of service levels to ensure common understanding of the trustwothiness of the processed data. This includes definition of the services, service levels, zones of trust to allow calculation of the trustworthiness when components from different vendors are used,

- Standardization of on board units that establish network connectivity and implement the mentioned protocols,

- Integration of on board units with the current assisting systems and definition of a common accepted user interface to advice the drivers.

Besides this, the functions and interaction mode of the current infrastructure need to be enhanced, the prediction systems and their runtime environment need to be developed and standardized, and the legal aspects, related to the current law and its advancements have to be considered.

Following this track ARTS can bring contribution to making the car traffic more efficient and sustainable and take a step towards developing smart cities. 
Acknowledgments: This research is partly supported by projects COST ARTS funded by EU Commission (www.cost-arts.org) and "AComIn: Advanced Computing for Innovation", Grant 316087, funded by the European Commission in FP7 Capacity (2012-2016).

\section{References}

1. Schlingensiepen, J. Korporative IT-Systemarchitektur zur Unterstuetzung unternehmensuebergreifender kooperativer Produktentwicklung. Integrierte Produktentwicklung, Teil Bd. 14. Otto-von-Guericke-Univ., Lehrstuhl fuer Maschinenbauinformatik, Magdeburg, 2008.

2. Smart Cities: Smart Cities - Ranking of European Medium-Sized Cities. October 2007.

3. United Nations Conference on Road Traffic: United Nations Conference on Road Traffic, Vienna, 7 October-8 November 1968. Final Act, Convention on Road Traffic, Convention on Road Signs and Signals. United Nations, New York, 1969.

4. Accenture: Building and Managing an Intelligent City. 2011, p. 44.

5. PublicPolicyEx: Smart Cities, Smart Europe. April 2014.

6. My Way: Multi-Modal Journey Planning Made Easy to Encourage the Use of Sustainable Modes of Transport.

7. L i t m a n, T. Ready or Waiting? Traffic Technology International. - ROW14, 2014.

8. W i 11 i a m s, B. Proof of Ownership. - Thinking Highways, Vol. 9, January 2014, No 1.

9. Castermans, J. The Future Is Green...eCoMove Green. - Thinking Highways, Vol. 9, March/April 2014, No 1.

10. AERIS: Latest Results for Aeris Program Show Fuel Benefits. April 2014.

11. B u 11 o u g h, J. D. Smart Illumination. Traffic Technology International, January 2014.

12. European Commission: Green Paper. Towards a New Culture for Urban Mobility. September 2007, p. 24.

13. United Nations Environment Programme: Towards a Green Economy: Pathways to Sustainable Development and Poverty Eradication. UNEP, 2011. ISBN 9789280731439.

14. European Commission: Communication from the Commission to the Council and the European Parliament on Thematic Strategy on the Urban Environment. January 2006, p. 12.

15. The European Parliament and the Council of the European Union: Directive 2010/40/EU of the European Parliament and of the Council of 7 July 2010 on the Framework for the Deployment of Intelligent Transport Systems in the Field of Road Transport and for Interfaces with Other Modes Of Transport. July 2010.

16. Urban ITS Expert Group: Guidelines for Its Deployment in Urban Areas - Multimodal Information. January 2013.

17. R i s t o, M., E. v a n B e r k u m. Connected Cruise Control (CCC). May 2013.

18. Schlingensiepen, J., R. Mehmood, F. C. Nemtan u, M. Niculescu. Increasing Sustainability of Road Transport in European Cities and Metropolitan Areas by Facilitating Autonomic Road Transport Systems (ARTS). - In: Sustainable Automotive Technologies 2013. Springer International Publishing, 2014, pp. 201-210.

19. Li a n g, J., K u m a r, R., X i, Y., R o s s, K. W. Pollution in P2P File Sharing Systems. - In: INFOCOM 2005, 24th Annual Joint Conference of the IEEE Computer and Communications Societies Proceedings IEEE, Vol. 2, March 2005, pp. 1174-1185.

20. Costa, C., A $1 \mathrm{meida}$, J.: Reputation Systems for Fighting Pollution in Peer-to-Peer File Sharing Systems. - In: Peer-to-Peer Computing, 2007 (P2P 2007), 7th IEEE International Conference, September 2007, pp. 53-60.

21. J e n s e n, C. T. SOA Design Principles for Dummies, Provided by Service Oriented Architecture (SOA): Simply Good Design, April 2014.

22. L a i r d, R. IBM White Paper - Key Entry Points for Implementing Service Oriented Architecture (SOA). Provided by Service Oriented Architecture (SOA): Simply Good Design, October 2013.

23. IP-KOM Consortium: IP-KOM-ÖV (Internet Protokoll basierte Kommunikation im Öffentlichen Verkehr). Internet Protocol-Based Communication in Public Transport, 2012. 